\title{
Prise en compte des médias sociaux dans la gestion des connaissances de l'entreprise
}

\author{
Étienne Deparis ${ }^{1}$, Gaëlle Lortal ${ }^{1}$, Marie-Hélène Abel ${ }^{2}$, \\ Juliette Mattioli $^{1}$
}

\author{
1. Laboratoire d'Analyse et de Raisonnement dans les Systèmes Complexes \\ Thales Research \& Technology \\ Palaiseau, France \\ etienne@depar.is,prenom.nom@thalesgroup.com \\ 2. UMR CNRS 7253 Heudiasyc \\ Université de Technologie de Compiègne \\ Compiègne, France \\ marie-helene.abel@utc.fr
}

\begin{abstract}
RÉSUMÉ. La popularité des réseaux sociaux en ligne amène les organisations à repenser leurs outils de collaboration. Se pose alors la difficulté de la capitalisation des ressources échangées. Nous présentons dans cet article la modélisation et le développement d'un écosystème numérique permettant la capitalisation à la fois des ressources issues d'un processus social et des ressources documentaires autour d'un référentiel partagé. Le modèle sous-tend aussi un processus de documentarisation adapté à ces ressources.

ABSTRACT. The wide adoption of social and connected tools in organizations leads them to think again about their behaviour and to consider the capitalization of the resources users can now produce with such tools, in the same way they capitalize other documentary resources. We present in this paper the model of a digital ecosystem which permits this capitalization of both documentary resources and those produced by a social platform. An ontology underlies the ecosystem enabling a shared capitalization and shared processes of documentarization.

MOTS-CLÉS : écosystème de connaissance, gestion de connaissance, ontologie, réseaux sociaux, web 2.0, entreprise 2.0, modélisation.

KEYWORDS: knowledge ecosystem, knowledge management, ontology, social network, web 2.0, enterprise 2.0, model.
\end{abstract}

DOI:10.3166/DN.17.2.55-79 (C) 2014 Lavoisier

Document numérique $-n^{\circ} 2 / 2014,55-79$ 


\section{Introduction}

Les médias sociaux drainent de plus en plus d'utilisateurs en ligne. Que ce soit à des fins de divertissement, d'information ou de veille personnelle, de nouvelles habitudes de partage et de production de ressources ont vu le jour. Conscientes des opportunités que peuvent présenter ces nouveaux usages dans leurs activités de collaboration ou de veille interne, les organisations s'intéressent de plus en plus aux médias sociaux. Leur déploiement sous la forme de solutions internes ou hébergées s'accélère. Cependant, la plupart des solutions actuelles ne permettent pas aux organisations une exploitation optimale. En effet, les solutions disponibles ne s'interfacent que rarement ou pauvrement avec les infrastructures déjà existantes. Cela se traduit par un éparpillement des informations au sein de différents outils ne communiquant pas entre eux.

Les organisations cherchent donc un moyen d'exploiter efficacement ces sources d'informations et de les faire cohabiter avec leurs bases de connaissances traditionnelles. Permettre aux organisations de mieux gérer l'ensemble de leurs ressources en les capitalisant autour d'un même référentiel quel que soit leur mode de création, est une clé essentielle d'amélioration des prises de décisions à tous les niveaux. Nous présentons dans cet article la réflexion qui nous a menés à penser un nouveau modèle théorique du document, modèle qui nous a ensuite guidés dans la réalisation d'une ontologie formalisant une plate-forme de gestion de connaissances et d'aide à la décision pour les organisations que nous présentons.

Nous expliquons dans la section 2 la pertinence d'intégrer des outils issus du web social au sein des SI des organisations. Après avoir défini ce qu'est un média social, nous précisons la raison pour laquelle ces derniers peuvent être favorables aux prises de décisions dans l'organisation. Nous présentons alors (section 3) un modèle théorique puis le modèle ontologique qui en est dérivé et qui permet de mettre en place un écosystème numérique pour la gestion de connaissances sociales pour l'aide à la décision au sein d'une organisation. Cette ontologie nous sert de base au développement d'un prototype d'aide à la décision que nous présentons dans la section 4 . Avant de conclure, la section 5 présente les résultats obtenus suite aux tests effectués à l'aide du prototype réalisé.

\section{Les interactions utilisateurs dans les écosystèmes numériques}

\subsection{Les personnes comme vecteur d'information}

La maîtrise de l'information est essentielle pour les organisations qui ont de tout temps essayé de la capter et de la capitaliser de manière à pouvoir la réutiliser le moment venu. Il s'agit d'un enjeu stratégique dans un monde en constante évolution (Ermine, 2000; Waltz, 2003). Maîtriser ses propres informations est un tremplin pour l'innovation et permet de perdurer sur un marché multi-concurrentiel. 
Toutes ces informations permettent à l'organisation de conserver un accès à son propre savoir, à la connaissance portée par ses collaborateurs. Cette connaissance peut se trouver sous deux formes : explicite ou implicite (Nonaka, Takeuchi, 1995). La maîtrise de la connaissance en entreprise passe par l'explicitation des connaissances implicites. Tout l'enjeu de cette explicitation est de trouver la transcription la plus adéquate possible de façon à éviter qu'une partie de ce savoir d'entreprise se perde si un collaborateur quitte l'organisation. Cette transcription passe encore aujourd'hui principalement par la rédaction de documents tels que des comptes-rendus de réunions, notes de positionnement, propositions, brevets, publications, etc. Le numérique en entreprise, bien qu'il ait aidé cette phase de transcription en apportant de nouveaux outils facilitant la prise de notes ou l'échange de documents, ne permet pas encore de pouvoir tout capitaliser.

Pourtant, la capitalisation des ressources échangées par les collaborateurs permettrait de mieux appréhender leurs champs d'expertises. De fait, cette problématique d'identification et de recherche d'experts au sein du tissu social d'une organisation est un vaste champ de recherche dont (Ehrlich et al., 2007) dressent un état de l'art, complété dans (DiMicco et al., 2009) par un état de l'art autour de la notion de people sensemaking. Les organisations proposent de longue date des outils de capitalisation documentaire fondés sur les techniques de documentation, voire de gestion électronique de documents (GED) plus automatisées. Cependant, avec ces systèmes, l'organisation ne peut capter ni retranscrire la connaissance implicite portée par les membres de l'organisation. Des outils améliorant le partage d'information et la collaboration, positionnant ainsi les personnes comme vecteur essentiel de l'information en entreprise sont mis en place dans la mouvance dite «Entreprise 2.0» (Boughzala, Dudezert, 2012). Au sein des organisations ayant déployé de tels outils en ligne, c'est tout un écosystème d'applications numériques qui vient servir les collaborateurs afin de les rapprocher numériquement.

Le terme d'écosystème numérique désigne, sur le web, un ensemble d'applications web interconnectées dont la survie individuelle dépend de la bonne santé (en termes de fréquentation, de revenus) de l'ensemble. Ce terme fait explicitement référence à la notion d'écosystème naturel ${ }^{1}$ qui désigne l'équilibre forgé par des êtres vivants visà-vis de leur milieu de manière à favoriser le maintien et le développement de la vie. Nous parlons par la suite d'écosystème applicatif quand nous évoquons un écosystème numérique observé du point de vue de son architecture d'applications. La notion d'écosystème de connaissance (Bray, 2007) est directement issue de celle d'écosystème numérique. Cette théorie s'appuie sur l'idée que l'innovation et l'apprentissage de nouvelles connaissances vont être drastiquement améliorés dans un environnement favorisant principalement les interactions humaines et plus particulièrement l'autoapprentissage d'une structure auto-organisée.

1. Terme utilisé pour la première fois, d'après Wikipedia, dans Tansley, Arthur George (1935). « The Use and Abuse of Vegetational Concepts and Terms », Ecology vol. 16 no 3 : 284-307. 
Cette approche a trouvé un écho avec le web 2.0, ce dernier permettant tout simplement la mise en pratique de ces idées via la mise à disposition d'outils autorisant cette auto-organisation des personnes et le libre échange de la connaissance. La base de cet écosystème est ainsi double : à une base documentaire courante dans l'écosystème des entreprises, on y adjoint des médias sociaux. Nous présentons ci-après ce que sont les réseaux sociaux et les médias sociaux définissant ce que nous considérons comme des connaissances sociales.

\subsection{Les réseaux et médias sociaux comme première brique d'un écosystème numérique}

Un réseau social est le nom donné à une représentation de structure sociale. Elle peut être constituée d'individus ou d'organisations et basée sur des relations d'amitiés, professionnelles ou commerciales (Barnes, 1954). La notion de réseau social dans le monde numérique est complexe. Si l'on se réfère exclusivement à la possibilité qu'offrent certains sites de créer des liens d'amitiés entre membres (Boyd, Ellison, 2008), les premiers d'entre eux sont apparus dans la seconde moitié des années 1990 avec classmates.com (1995), sixdegrees.com (1996), Asian Avenue et Black Planet (1999), suivis plus récemment de LinkedIn et MySpace (2003), Orkut et Facebook (2004). Par la capacité qu'ils ont à concevoir des microsociétés autour de thématiques, les moyens de communication électroniques qui ont explosé dans les années 1980 (mails, groupes usenet, puis blogs, microblogs, etc.) peuvent être considérés comme des réseaux sociaux. La façon même que va avoir un acteur de s'adresser à différents membres de groupes variés va créer une structure sociale forte sous-jacente à l'outil électronique (Fisher et al., 2006).

Dans (Seufert et al., 1999), est soulignée l'importance de concilier réseau social et gestion de la connaissance. Ils rappellent ainsi combien les entreprises se regroupent aujourd'hui préférentiellement en partenariats industriels et ne travaillent plus seules. La gestion de la connaissance au sein de tels partenariats va devoir passer avant tout par la reconnaissance en interne de réseaux sociaux informels (Morgan, 1986 et Sandner 1990 dans (Seufert et al., 1999)), souvent constitués de personnes partageant des affinités fortes (McPherson et al., 2001). Les réseaux sociaux numériques sont souvent considérés comme potentiellement nuisibles à la productivité d'une équipe, en autorisant l'utilisation des ressources de l'organisation à des fins personnelles ou simplement l'oisiveté (Shirky, 2008 ; Ferreira, Plessis, 2009). Néanmoins, ils peuvent agir bénéfiquement pour l'entreprise en facilitant les interactions à distance entre collaborateurs et en améliorant le partage d'informations et de connaissances au sein de l'organisation.

Pour désigner une application fondée sur un réseau social et permettant une publication, nous utilisons le terme de média social. Pour désigner les données provenant des médias sociaux nous utilisons le terme de données sociales. Les données sociales peuvent être de formes textuelle ou iconique. Elles sont matérialisées par le contenu 
d'un blog ou un wiki, ou un log d'activité, aussi bien que par une balise d'étiquetage ou graphe d'interactions d'utilisateurs (Lortal et al., 2013).

L'apparition d'outils tels que les wiki (1995) ou les plates-formes de blog (blogger, en 1999) a transformé le web, le faisant passer d'un statut de cinquième média à celui plate-forme sur laquelle le visiteur est appelé à ajouter ses propres créations, le contenu de son choix. Cette révolution numérique, soutenue par l'usage de médias sociaux, a pris le nom de web 2.0 (O'Reilly, 2009). Elle a servi de catalyseur à de nombreux nouveaux usages vis-à-vis de l'information. Les informations ne sont plus seulement accessibles (au travers d'encyclopédie, de bibliothèques, etc.), elles sont avant tout dynamiques, modifiables, incrémentales. Le consommateur est devenu acteur. Ces changements se sont répercutés dans bien des domaines et en particulier dans la culture des entreprises orientées Information Technology (IT). Une structure parallèle à la structure hiérarchisée apparait au profit d'une transversalité plus grande qui conduit à une accélération des échanges et de nouveaux modes d'innovations par une synergie sociale. Les échanges s'effectuent dans les organisations à l'aide d'offres intégrées et bien souvent gratuites (Google Docs, Doodle, Trello, etc.) qui entrent en compétition directe avec les propres outils de l'organisation. Ces services sont souvent vus comme vieillissants et abandonnés au profit de plates-formes web plus modernes. Devant l'implantation toujours plus forte de ces nouvelles plates-formes au sein de leurs équipes de travail, les organisations réfléchissent de plus en plus à comment faire cohabiter ces nouveaux outils avec leur système d'information historique. Le flux principal d'information quittant ainsi les entreprises n'est pas lié à ses documents métiers (brevets, comptes-rendus, etc.). Ces derniers sont toujours capitalisés au sein de bases de connaissance internes. Les connaissances perdues sont liées aux interactions des membres de l'organisation, aux échanges d'informations auxquels ils se livrent quotidiennement. Ainsi, bien qu'identifiée, cette source de connaissance échappe encore aux organisations. Il apparaît alors nécessaire que ces dernières déploient au sein de leur SI un écosystème de connaissance permettant à ses membres de goûter aux mêmes fonctionnalités que celles qu'ils plébiscitent sur le web, tout en permettant la bonne capitalisation des données échangées ou produites au sein de ces applications web.

Il apparaît crucial pour les organisations de mettre en avant leur réseau social au travers d'applications facilitant la collaboration et l'échange rapide d'informations afin de pouvoir conserver toutes ces données et en faire émerger des tendances et in fine de l'innovation (Tsai, Ghoshal, 1998). Nous allons présenter dans la section suivante l'approche théorique que nous proposons, permettant d'associer au sein d'une même base de connaissances des ressources issues tant du monde documentaire que du web social. Ce modèle théorique est le fondement d'une ontologie originale formalisant un écosystème applicatif autour d'une plate-forme de gestion de connaissances tenant compte des dimensions sociale et documentaire des organisations et de briques venant outiller les décideurs des organisations en puisant et présentant d'une manière adéquate les ressources enregistrées dans la base de connaissances. 


\section{Modèle théorique et ontologique}

\subsection{Modèle théorique du document}

La modélisation de notre écosystème numérique de connaissance est parti de ce simple constat : les échanges ayant lieu au sein des plates-formes sociales peuvent représenter de la connaissance pour l'organisation. Cette connaissance est peu prise en compte aujourd'hui. Ces connaissances peuvent servir dans le cadre des processus de décisions, très courants dans les organisations.

Les organisations utilisent des systèmes interactifs d'aide à la décision (SIAD) pour encadrer leurs processus de décision. Ces SIAD servent les décideurs de l'organisation en leur permettant d'identifier les différents choix de réponses possibles à un problème en puisant dans les bases de connaissances de l'organisation. Il ne s'agit pourtant matériellement que de bases de données stockant des éléments qui, pris séparément, sont tout à fait abscons. L'intérêt des SIAD et de leurs bases de connaissances est double. Il s'agit d'une part de conserver l'information permettant de présenter ces données à un décideur de l'organisation de telle manière qu'il en tire la connaissance voulue par la personne ayant organisé l'enregistrement des données en question. Il s'agit d'autre part de profiter de la manne que constitue l'ensemble des données enregistrées pour présenter des données liées ensemble de manière originale à un décideur. Ces liens entre données sont inférés à l'aide de règles renseignées par un décideur ou un expert de l'organisation et lui permettent ainsi de tirer de nouvelles connaissances des données enregistrées dans la base de connaissances.

Les principaux éléments constitutifs de ces bases de connaissances sont avant tout des données documentaires ajoutées par les membres de l'organisation. La définition de document dans le monde numérique peut souffrir d'un positionnement relativement flou qu'il convient de fixer, du moins pour nos besoins propres. Cette définition doit, de plus, permettre de faire le lien entre la notion de document numérique et celle de connaissance. S'il ne semble s'agir ici que d'un problème autour du sens du mot document, nous souhaitons ouvrir ce positionnement pour considérer également les productions effectuées au sein des médias sociaux. Ces productions peuvent être vues comme les traces d'une activité sociale. Elles semblent alors, à ce titre, entrer dans la définition de document numérique de (Pédauque, 2003), où le document est vu comme medium. Cependant, les médias sociaux dans leurs formes et leurs usages exposent les objets numériques considérés à une grande dynamique. Les productions des médias sociaux peuvent alors être vues comme des ressources, au sens de (LainéCruzel, 2004). Dans cette optique, elles seraient incompatibles avec un stockage au sein d'une base de connaissances, qui demanderait une stabilité propre à l'archivage.

Nous faisons évoluer ce positionnement de (Lainé-Cruzel, 2004), en particulier en revisitant la notion de ressource dans le monde numérique et en adoptant la définition de (Prax, 2012). La figure 1 illustre notre modèle théorique original. Dans le monde numérique, une ressource est la matérialisation numérique d'un ensemble d'informations (Prax, 2012). 


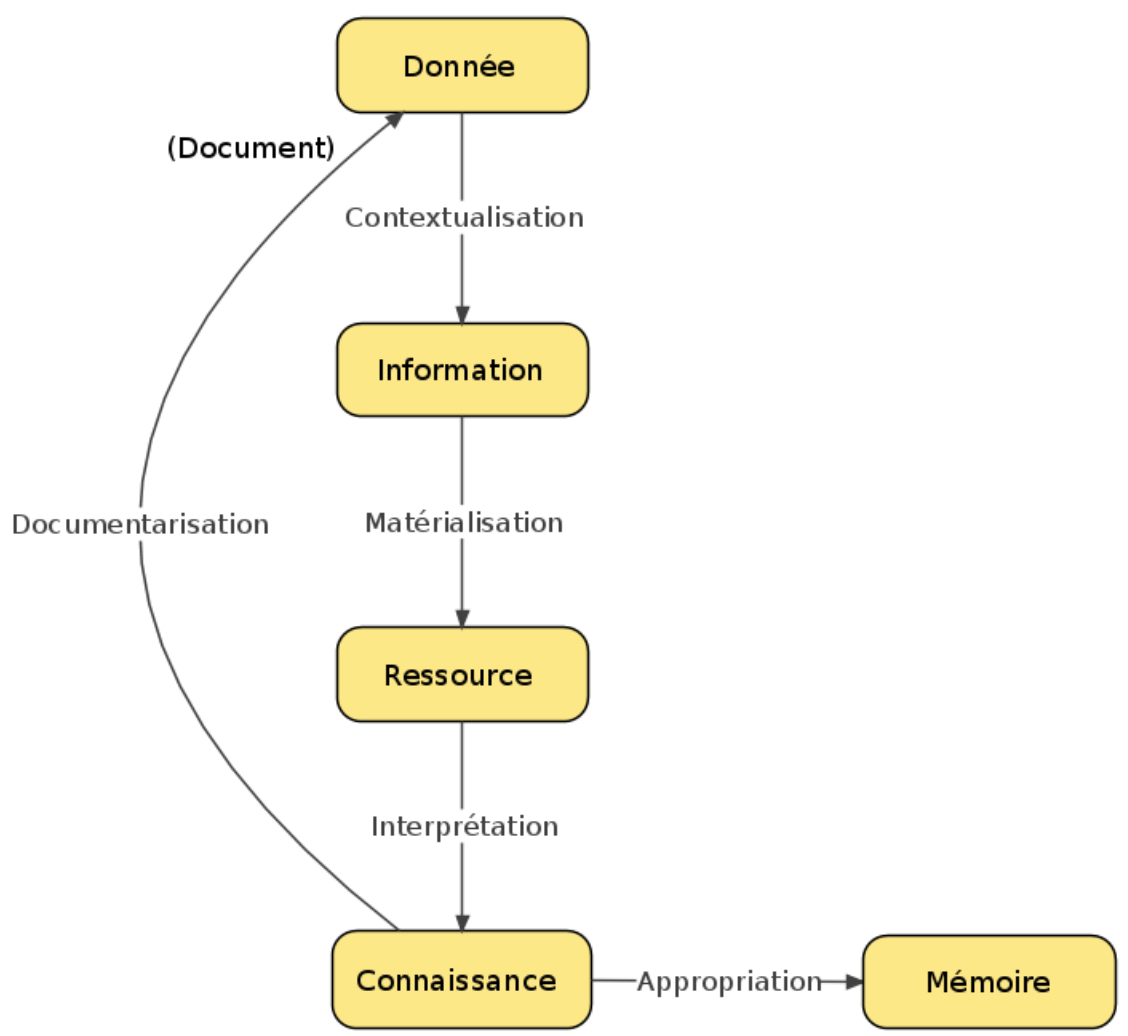

Figure 1. Schéma de situation de la notion de ressource

En effet, l'utilisation d'un environnement numérique peut cacher aux utilisateurs les niveaux d'accès aux données et aux informations. À la recherche d'une information dans le système, l'utilisateur se verra proposer en réponse un ensemble de données re-contextualisées (grâce à la structure de la base de données et les liens placés entre les données y étant sauvegardées) et mises en forme (selon les spécifications implémentées dans le système utilisé). Nous appelons ces résultats des ressources. Notre définition de ressource s'éloigne de celle de (Lainé-Cruzel, 2004) pour revenir vers le point de vue du «document comme forme » de (Pédauque, 2003).

Dans l'environnement numérique, les ressources s'affichent à l'écran de l'utilisateur et n'ont aucune autre mission que de lui présenter de l'information. L'utilisateur peut alors, dans un acte volontaire d'appropriation de la ressource, la percevoir en tant que document. Ce phénomène d'appropriation se rapproche du point de vue du document comme signe de (Pédauque, 2003).

Notre définition procède donc à l'identification de trois étapes intermédiaires entre la notion de donnée et celle de connaissance 1. En suivant le schéma, on peut lire 
que la contextualisation d'une donnée stockée dans le SI produit une information. Cette information, ou ces informations s'il y a composition, peuvent être matérialisées de différentes façon selon ce que l'utilisateur souhaite appliquer comme vue pour se l'approprier. L'utilisateur a alors accès à une ressource qu'il interprète selon ses activités et ses buts. L'appropriation d'une connaissance par un individu se décline selon qu'il la conserve simplement en mémoire ou qu'il souhaite la repartager, enregistrant dans le SI de son organisation la ressource support de cette connaissance. Nous considérons que l'interprétation des informations mises en forme dans une ressource est nécessaire à la découverte de nouvelles connaissances et fait partie intégrante du phénomène d'appropriation de la ressource en document. Nous parlons dans ce dernier cas de documentarisation, c'est-à-dire de l'action selon laquelle un utilisateur ayant interprété une ressource et souhaitant la conserver sous cette forme, l'enregistre intentionnellement dans la base de connaissances de l'organisation. Ce document peut désormais être utilisé dans le SI comme n'importe quelle autre donnée.

\subsection{L'ontologie memorae-core 2}

Nos travaux s'intègrent en partie dans l'approche MEMORAe. Celle-ci propose un modèle sémantique et une plate-forme de collaboration et de gestion de connaissances pour les organisations. Cette approche se positionne sur le même secteur que la plupart des écosystèmes numériques de connaissances actuels tels que SharePoint, BlueKiwi, Atlassian, Yammer, Pearltrees, etc. Cependant, la capitalisation de ressources hétérogènes autour d'un référentiel sémantique présenté à l'utilisateur sous la forme d'une cartographie de concepts distingue l'approche MEMORAe de ces produits.

En effet, afin de permettre la prise en compte homogène de tous les types de ressources, qu'elles soient issues d'un processus social au sein d'une application web ou simplement d'une base de connaissance de l'organisation, notre écosystème s'organise autour du pivot représenté par un référentiel unique et partagé grâce auquel toutes les ressources telles que messages de forums, pages de wikis, minimessages, etc. seront capitalisées. C'est également grâce à ce même référentiel que les membres de l'organisation vont pouvoir être caractérisés, qualifiés.

Nous nous attachons ici à présenter les travaux que nous avons menés pour modéliser la structure sociale et fonctionnelle d'un tel écosystème et les ressources y étant échangées et non la modélisation du référentiel métier de l'organisation, réalisée par ailleurs à l'aide d'une ontologie de domaine par les experts de cette même organisation. Nos travaux étendent le modèle et les fonctionnalités développés précédemment, telles que le wiki, les forums, ou encore les agendas partagés (Leblanc, Abel, 2008 ; 2010). Cette évolution du modèle a principalement consisté à mieux considérer la dimension sociale d'une organisation à l'aide de standards actuels du web sémantique que nous avons intégré au sein d'une nouvelle ontologie que nous avons appelée 
memorae-core $2^{2}$. Nous présentons, dans la suite, différentes particularités de cette ontologie et les standards utilisés dans sa conception.

\subsubsection{Représentation du graphe social}

Le projet FoaF (Brickley, Miller, 2010) est l'une des plus anciennes initiatives du web sémantique cherchant à définir une communauté humaine. Avec une approche très simple, il s'est progressivement imposé comme un standard de fait que l'on retrouve aujourd'hui à la base de beaucoup d'autres projets joignant sémantique et social. C'est le cas par exemple du projet SIOC (Breslin et al., 2009; Bojārs, Breslin, 2010) qui spécialise les concepts définis dans FoaF afin de décrire des communautés en ligne et quelques ressources (forum, wiki, etc.) autour desquelles elles peuvent se fédérer.

Leur large adoption dans la communauté et la possibilité de pouvoir décrire à la fois le côté humain (grâce à la classe foaf : Person) et les comptes utilisateurs (avec la classe sioc: UserAccount) nous ont conduit à fonder notre représentation des individus d'un graphe social sur ces ontologies.

Au sein d'une organisation, les collaborateurs sont généralement distribués en équipes définies par une entité hiérarchique supérieure. Ces équipes institutionnelles constituent une partie de la structure sociale de l'organisation.

Une part des activités de socialisation des collaborateurs d'une organisation passe également par la rencontre, l'échange d'informations avec des individus ne faisant pas partie de leur équipe institutionnelle. Il est donc nécessaire de tenir compte dans la modélisation de la structure sociale des liens entre personnes, comparable à des groupes d'individus librement constitués.

Nous avons fait le choix de modéliser la structure sociale d'une organisation à partir de la définition des équipes institutionnelles et dynamiques. Pour ce faire, nous avons décidé également d'utiliser les standards FoaF et SIOC. Ces deux ontologies nous permettent de couvrir une partie de nos besoins de modélisation des équipes ou groupes d'individus. Nous constatons néanmoins les manques suivants :

1. l'absence de différenciation des types de groupes d'individus ;

2. l'absence de moyen de représenter des liens inter-organisationnels, typiquement les liens d'imbrications des différents services et divisions d'une grande organisation.

Pour cela nous spécialisons la notion de groupe proposée par FoaF selon les caractéristiques suivantes :

- Un groupe institutionnel est créé par une entité hiérarchique et regroupe différents collaborateurs autour d'une mission déterminée. Il s'agit typiquement des équipes de travail au sein des organisations.

2. Cette ontologie est disponible sous la forme d'un fichier au format turtle à l'adresse http://www.hds.utc .fr/memorae/core2.ttl 
- Un groupe dynamique est créé à l'instigation des membres de l'organisation sans validation hiérarchique. Il peut s'agir de communautés d'intérêt autour d'un sujet précis, d'une personne ou des deux. L'ouverture d'un tel groupe aux autres membres de l'organisation peut être restreinte, dans le cadre de groupes privés.

\subsubsection{Représentation des ressources}

Il est important de représenter à la fois les ressources documentaires et celles issues d'interactions sociales en ligne. Cette modélisation doit permettre de lier ces différentes ressources à un même référentiel. De même que pour les relations sociales, nous avons choisi les ontologies FoaF et SIOC pour représenter les ressources issues d'un processus social.

Pour représenter les ressources documentaires classiques, nous nous sommes appuyés sur l'ontologie BIBO (D'Arcus, Giasson, 2009). Celle-ci permet de décrire de manière presque exhaustive tout contenu bibliographique très simplement. Elle tend en effet à suivre les mêmes règles de nommage que celles employées avec Bibtex. La conversion d'une bibliographie accessible au format Bibtex selon le vocabulaire sémantique de BIBO est ainsi facilitée.

Nous avons cependant précisé qu'une ressource pouvait être simple ou composée. En effet une ressource peut être le résultat de l'association d'autres ressources. C'est le cas par exemple des réponses aux questions du forum. Chaque réponse ou question peut se suffire à elle-même. Le fil de messages apporte alors un éclairage particulier supplémentaire.

\subsubsection{Lien de thématique}

Nous avons identifié que l'un des principaux problèmes des systèmes de capitalisation actuels est de ne pas utiliser un même référentiel pour la description des ressources documentaires et celles issues d'interactions sociales. D'un point de vue ontologique nous cherchons donc une relation entre une ressource (foaf : Document, bibo: Article, etc.) et un concept préalablement défini (owl:Thing), voire une classe non instanciée provenant d'une ontologie de domaine, par exemple un référentiel partagé au sein d'une organisation.

Il nous a pour cela semblé intéressant de concevoir une nouvelle classe mc2: Indexkey. Cette classe nous permet, en plus d'associer une ressource à un concept défini par ailleurs dans un référentiel partagé, de définir que cette indexation s'effectue exclusivement pour un espace partagé appartenant à un groupe particulier du système. En effet, dans notre modélisation, chaque groupe se voit affecter un unique espace de partage. Cet espace unique permet aux membres du groupe de s'échanger des ressources entre eux, sans que ces dernières ne soient visibles des autres utilisateurs de la plate-forme. 


\section{Présentation de l'écosystème fondé sur notre modélisation}

La plate-forme actuelle se compose de plusieurs briques logicielles rassemblées sous l'appellation SIDEKICK ${ }^{3}$. L'objectif de cet écosystème d'applications est d'offrir une solution complète au service des organisations, allant d'un environnement de gestion de connaissances pour les membres de l'organisation au système d'analyse des données échangées au sein de l'environnement de gestion de connaissances pour les décideurs. Les trois briques essentielles de cet écosystème feront l'objet, pour chacune d'entre elles, d'une section particulière et sont :

- la brique de gestion de connaissances;

- la brique inférentielle ;

- la brique de visualisation.

L'architecture générale de cette plate-forme est illustrée en figure 2.

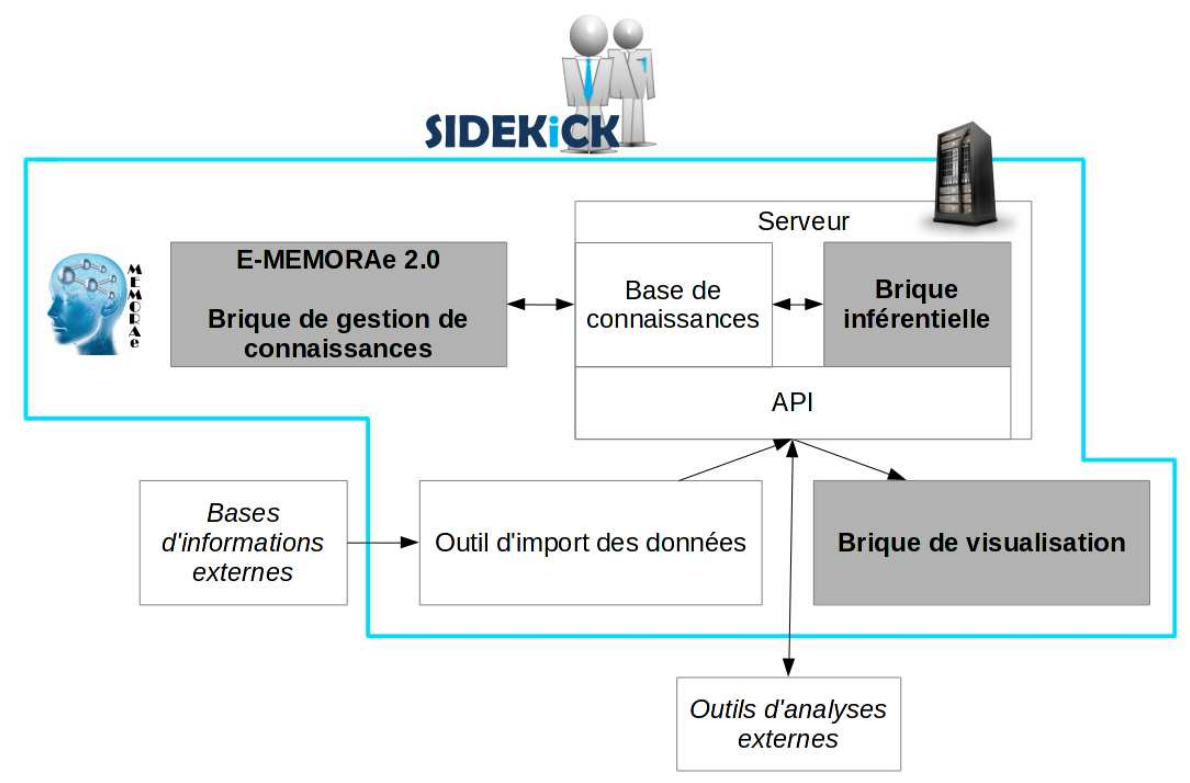

Figure 2. Architecture globale de notre prototype d'écosystème

\subsection{Brique de gestion de connaissances}

La brique de gestion de connaissances est une évolution de la plate-forme EMEMORAe 2.0 de l'Université de Technologie de Compiègne. Elle a été dévelop-

3. Le nom de cette plate-forme est un acronyme pour Software for socIal Decision support Enabled by Knowledge Interpreted from Collected Key 
pée avec le toolkit Adobe Flex. L'intérêt de cette brique réside dans son intégration de différents outils sociaux tels qu'un forum ou un wiki, permettant aux membres de l'organisation de capitaliser leurs échanges de la même manière que les autres ressources documentaires, selon le même référentiel.

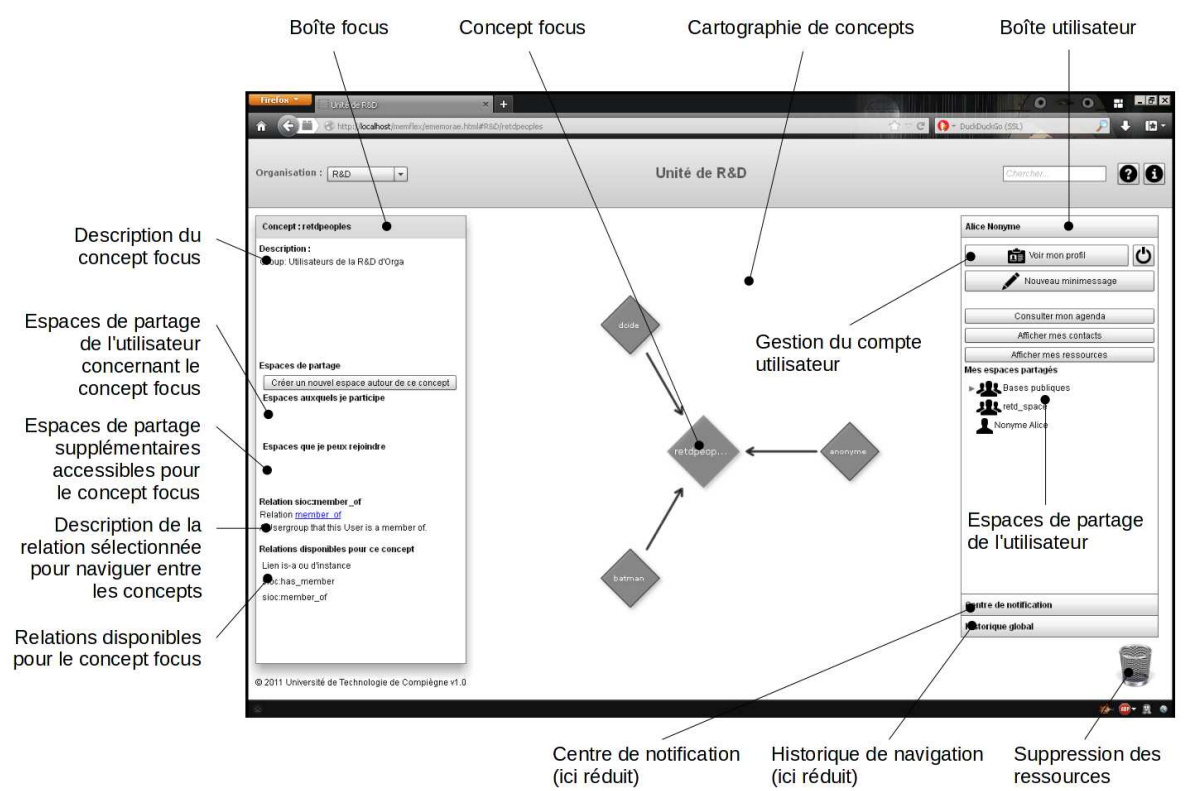

Figure 3. Capture d'écran de l'environnement E-MEMORAe 2.0

La figure 3 présente une capture d'écran de cette brique. Le référentiel commun utilisé pour indexer les ressources y prend la forme d'une cartographie de concepts affichée en permanence de manière centrale à l'utilisateur. Autour de cette cartographie s'articulent deux boîtes destinées à présenter les informations concernant le focus de la carte ou celles de l'utilisateur courant.

La boîte utilisateur (à droite sur la figure 3) contient principalement le bloc de commandes permettant à l'utilisateur de modifier son profil ou se déconnecter et le bloc d'accès aux différents espaces de partage auxquels il participe.

En effet, la brique permet la gestion de groupes d'utilisateurs qui autorise ainsi les organisations à insérer en quelque sorte leur structure hiérarchique au travers des groupes institutionnels. Mais surtout, la brique permet aux utilisateurs de se rassembler en groupes libres autour d'une thématique issue du référentiel commun.

Chacun des groupes est lié à un espace de partage unique et ce sont donc ces derniers qui sont affichés dans le bloc d'accès aux différents espaces de partage. Lorsqu'ils sont ouverts, les espaces de partage permettent à l'utilisateur d'accéder à ses ressources. Le contenu des espaces de partage est contextuel et n'affiche que les ressources indexées pour le concept focus. Ainsi, l'accès aux ressources ne se fait pas directement suite à une recherche sur un moteur, mais suite à la navigation volontaire 
de l'utilisateur au sein d'une cartographie de concept, le référentiel commun de son organisation. Cette vue décentralisée de l'utilisateur sur les ressources, toujours fondée sur le concept focus, permet de n'afficher à l'utilisateur que les informations qui l'intéressent vraiment, quel que soit leur type.

Le fait de restreindre l'affichage des ressources au concept focus peut s'illustrer avec notre implémentation d'un système de minimessage (microblogging). En effet, nous associons directement les hashtags présents dans les minimessages aux concepts de l'ontologie et capitalisons ces minimessages sur ces concepts. Ils ne deviennent alors visibles que lorsque ces concepts ont le focus. Les utilisateurs peuvent ajouter des instances au référentiel commun, mais la structure de base n'est accessible que du système. L'utilisation des mentions dans les minimessages permet à leurs expéditeurs d'informer rapidement un autre utilisateur. Dans l'exemple suivant : «@mhabel http://www.hds.utc.fr/memorae est une plate-forme intéressante \#km \#social_network » l'utilisatrice mhabel se verra informée par une notification de l'ajout de ce minimessage. Cette notification apparaîtra dans le centre de notification visible sur la figure 3 .

La boîte focus (à gauche sur la figure 3) contient les informations concernant le concept focus actuellement affiché au centre de la cartographie. À l'instar des espaces de partage, la boîte focus est donc contextuelle. Outre l'affichage du label et de la description du concept focus, la boîte focus donne accès aux différentes relations disponibles pour ce concept. La cartographie de concepts étant en fait une représentation d'une ontologie écrite en OWL, ces différentes relations sont simplement les Object Properties associées à ce type de concept. Le fait de cliquer sur le nom d'une de ces relations recharge la cartographie centrale pour ne plus montrer que les relations de ce type. La partie centrale de la boîte focus permet d'afficher les espaces de partage concernant le concept focus, c'est-à-dire les espaces de partage liés aux groupes créés autour de ce concept. Elle permet également de créer un groupe libre souhaitant échanger autour de ce concept. À sa création, ce groupe est constitué d'un seul membre : l'utilisateur créateur. Les autres membres pourront le rejoindre au fil de l'eau.

\subsection{Brique inférentielle}

Cette brique doit permettre de calculer le graphe (fait de nœuds et de liens) du réseau social de collaboration des différentes organisations dont les informations ont été enregistrées dans la base de connaissances. Chaque nœud de ce graphe représente un partenaire différent, tandis qu'un lien entre deux nœuds doit représenter la force de la relation unissant les deux partenaires.

Le calcul de la force des relations est effectué à l'aide d'une version modifiée de l'algorithme d'edgerank utilisé sur Facebook, dont nous rappelons l'expression originale dans la formule (1). Cette formule permet de ranger les différents objets devant apparaître sur le wall d'un utilisateur A en fonction des paramètres suivants :

- $u_{e}$ degré d'affinité entre l'utilisateur A et l'auteur de l'objet considéré ; 
- $w_{e}$ facteur de pondération du score final en fonction du type d'objet considéré ;

$-d_{e}$ facteur de pondération du score final en fonction de l'ancienneté de l'objet considéré.

$$
\sum_{\text {edges } e} u_{e} w_{e} d_{e}
$$

À la différence du graphe social de Facebook, composé d'utilisateurs s'organisant autour d'objets de différents types (page web, messages personnels, liens, photographies, commentaires, etc.), notre graphe est lui composé d'organisations et des liens qu'elles entretiennent au travers de publications communes, de comptes-rendus, de brevets, etc. Du fait de nos données, les paramètres de la formules vont avoir, dans notre implémentation, les significations suivantes (où $A$ et $B$ désignent deux entités ayant collaboré au travers de différents edges e représentant donc les différents liens ou actes de collaboration passés) :

- $u_{e}$ est le coefficient de corrélation d'un acte de collaboration à un certain concept $k$ du référentiel commun, donné par le décideur. Dans le cadre d'une analyse générique, c'est-à-dire lorsqu'aucun concept n'est donné par le décideur, ce coefficient prend simplement la valeur 1. Dans le cadre de l'analyse des collaborations portant sur un concept $k$, ce coefficient permet de mettre en valeur les liens impliquant effectivement ce concept et de mettre en retrait les autres liens. Le coefficient est alors calculé à l'aide de la formule (2). Dans cette formule, nous posons $K$ comme étant l'ensemble des concepts $k_{i}$ utilisés pour étiqueter la collaboration $l$ étudiée. Nous posons $k i_{\text {match }}$ comme ayant une valeur de 1 si $k_{i}=k$ et 0 sinon. Nous permettons au décideur de régler finement l'importance relative qu'il veut donner à la correspondance thématique à l'aide de la constante d'appréciation $a_{k}$ qui ne sera prise en compte que dans le cas des collaborations portant sur le concept $k$.

$$
u_{e}=\sum_{k_{i} \in K} \frac{k i_{\text {match }} a_{k}}{\operatorname{Card}(K)}
$$

- $w_{e}$ donne l'importance relative du type de collaboration (publication, brevet, etc.). Nous avons fixé ces valeurs selon le type de publication dans le code source de notre prototype. Ces dernières sont bien sûr modifiables en fonction de l'importance relative que l'on souhaite donner à un type de collaboration.

- $d_{e}$ est le coefficient de dépréciation temporel d'un acte de collaboration défini par la formule (3). Pour ce calcul, nous posons $t 1$ comme étant la date de fin de cette collaboration et $t 2$ la date courante au moment du calcul (date pouvant varier pour mesurer l'évolution de la force d'un lien de collaboration à travers le temps. Nous autorisons le décideur à influer sur le coefficient de dépréciation temporel en lui permettant de fixer la constante $c_{t}$. Cela permet de contrôler l'influence du temps vis-à-vis des autres coefficients de la formule (1). 


$$
d_{e}=\frac{c_{t}}{(t 2-t 1)+1}
$$

Ainsi, notre formule développée finale, notée $S_{A B}$ (pour Score de la collaboration entre $\mathrm{A}$ et $\mathrm{B}$ ), peut prendre deux formes selon que nous souhaitons procéder à un classement des relations entre acteurs sur un concept en particulier (formule (4)) ou de manière générique (formule (5)).

$$
\begin{gathered}
S_{A B}=\sum_{\text {edges } e} \overbrace{\left(\sum_{k_{i} \in K} \frac{k i_{\text {match }} a_{k}}{\operatorname{Card}(K)}\right)}^{u_{e}} \times w_{e} \times \overbrace{\frac{c_{t}}{(t 2-t 1)+1}}^{d_{e}} \\
S_{A B}=\sum_{\text {edges } e} w_{e} \times \overbrace{\frac{c_{t}}{(t 2-t 1)+1}}^{d_{e}}
\end{gathered}
$$

Le calcul d'Edgerank se prête bien pour le calcul de l'importance d'un lien pouvant effectivement exister entre deux entités. Il requiert néanmoins l'existence d'une trace de collaboration entre les deux entités considérées, ce qui n'est bien sûr plus possible lorsque l'on cherche à évaluer la proximité thématique de deux entités n'ayant encore jamais collaboré.

Nous exprimons cette proximité thématique sous la forme de liens potentiels unissant deux entités. Ces liens vont avoir une importance plus ou moins grande en fonction du nombre de concepts partagés par les deux entités et la proximité temporelle de leurs travaux sur ces concepts.

Pour un concept $k$ et deux entités $A$ et $B$ donnés, nous définissons ces liens potentiels à l'aide de leur score noté $S P_{A B}$ (pour Score de la collaboration Potentielle entre A et $B$ ) selon la formule (6), qui utilise les paramètres suivants :

- la durée des dernières périodes de collaboration de $A$ et $B$ sur $k$;

- le type des dernières périodes de collaboration de $A$ et $B$ sur $k$;

- la durée de travail parallèle, ou recouvrement, sur $k$ de $A$ et $B$, au cours de leurs derniers travaux respectifs sur $k$, si elle existe.

- l'ancienneté de cette période de recouvrement, si elle existe.

$$
S P_{A B}=\frac{g p}{p p} \times \frac{\text { recouvrement }}{\text { ancienneté }}
$$

La formule (6) finale requiert de comparer les deux durées de travaux de $A$ et $B$ sur $k$, afin d'identifier la durée de travail la plus longue. Nous utilisons alors les termes de $g p$ (grande période) et $p p$ (petite période). Ces dernières peuvent concerner indifféremment $A$ ou $B$. 


\subsection{Brique de visualisation}

Jusqu'à présent nous avons pu voir de quelle manière les utilisateurs de notre prototype pouvaient peupler une base de connaissances à l'aide de la brique de gestion de connaissances (section 4.1). Notre positionnement théorique stipule que nous pouvons considérer cette base de connaissances comme une base de données stockant à la fois des données et leurs contextes, permettant de les re-matérialiser sous la forme de ressources. La brique inférentielle présentée précédemment (section 4.2) se positionne avant la phase de matérialisation des données. Elle ne traite en effet que des informations, c'est-à-dire des données re-contextualisées issues de la base de connaissances. Le but de ce traitement, comme nous l'avons vu, est de trouver de nouvelles relations entre les informations déjà enregistrées dans la base. La brique de visualisation que nous avons ajoutée à notre prototype permet la matérialisation de ces nouvelles informations et leur consultation par les décideurs d'une organisation. Cette brique de visualisation, conçue comme une application web intégrée à l'écosystème applicatif que forme notre prototype, a été réalisée à l'aide de la bibliothèque javascript $\mathrm{d} 3 \mathrm{j}$. Elle présente au sein d'un tableau de bord unifié différents cadrans, dont ceux que nous verrons plus précisément dans cet article :

- le graphe des collaborations passées ou possibles des différentes entités enregistrées dans la base de connaissances de l'organisation;

- un lien vers l'outil de génération de rapport de proposition de consortiums.

La figure 4 présente la vue générale de la brique de visualisation au démarrage. À ce moment là, seul le graphe de collaboration est visible, les autres cadrans n'étant ouverts qu'au besoin. Ils viennent alors se positionner au dessus du graphe de collaboration. Chacun de ces cadrans possède des outils permettant aux décideurs d'interagir avec les informations matérialisées sous leurs yeux, de les mettre en forme selon leurs préférences et éventuellement de les exporter. Ce faisant, les décideurs s'approprient les ressources affichées à l'écran, processus via lequel ils vont pouvoir tirer de nouvelles connaissances qu'ils pourront capitaliser au sein de la base de connaissances de l'organisation en y sauvegardant le document, fruit de leur appropriation des ressources présentées.

Le cadran de graphe des collaborations passées prend la forme du graphique visible sur la figure 4. Ce dernier a été réalisé après traitement d'un ensemble d'articles ayant été publiés au sein de la communauté scientifique d'ingénierie des connaissances (IC) et ajouté à la base de connaissances du système. Ces articles sont issus de la base librement consultable HAL et sont au nombre de 206, couvrant une période allant de 1998 à 2002. La vue actuelle du graphe de collaboration peut être exportée à tout moment vers le logiciel Gephi.

Les informations utilisées pour générer le graphe de la figure 4 ont été calculées par la brique inférentielle au fur et à mesure de l'ajout de nouvelles informations dans la base de connaissances. Chaque nœud du graphe représente une entité et la taille des liens pleins reliant les nœuds deux à deux est définie par notre formule du Edge- 


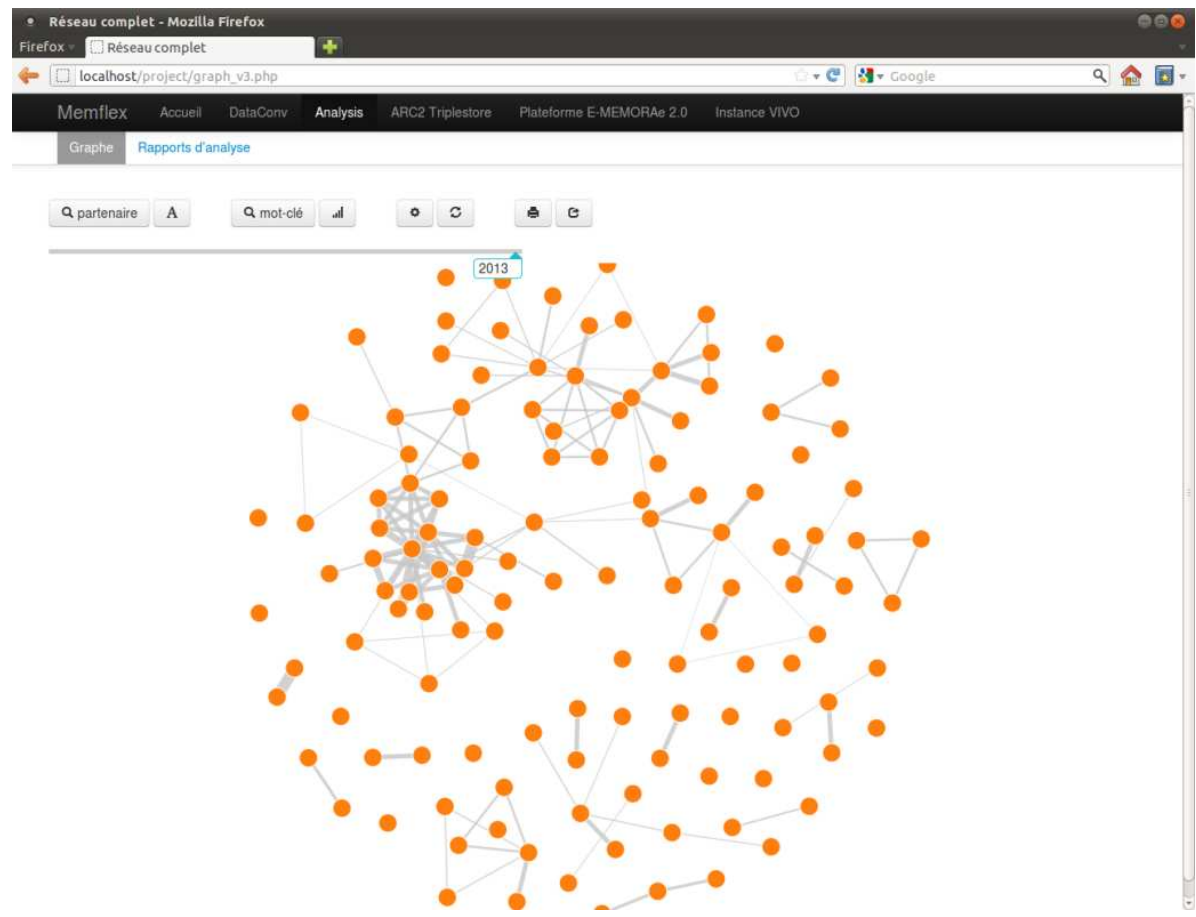

Figure 4. Vue générale de la brique de visualisation

rank. L'algorithme de Edgerank a permis de calculer l'importance des liens factuels ou potentiels entre chaque organisation du réseau. Les liens potentiels apparaissent en pointillés et leur taille est calculée à l'aide de la formule (6) section précédente.

Le cadran de visualisation du graphe des collaborations permet également d'observer l'évolution de ce graphe au travers des années. La sélection de l'année s'effectue à l'aide de la tirette de sélection d'année du graphe de collaboration (visible sur la figure 4 au-dessus du réseau de collaborations).

La sélection d'une entité au sein du graphe de collaboration permet à un décideur d'accéder à l'outil de génération de rapports de proposition de consortiums. Cet outil se sert à la fois de règles de modélisation d'appel à projet décrites dans la base de connaissances (Deparis, 2013) et du même calcul de la force des liens avérés ou potentiels entre deux entités du graphe de collaboration. Il s'agit de pouvoir générer un nouveau type de ressources à partir d'informations extraites de la base de connaissances.

Après avoir sélectionné le type d'appel à projet à surveiller et éventuellement réduit l'observation à un ou plusieurs concepts précis du référentiel partagé, le décideur lance la génération du rapport. Ce dernier prend la forme d'une suite de paragraphes. 
Chaque paragraphe d'un rapport est dédié à un type d'appel à projet et un concept particulier, parmi ceux sélectionnés. Le paragraphe rappelle tout d'abord les règles à respecter pour valider le montage du consortium et liste ensuite les entités issues du graphe de collaboration qui semblent pertinentes pour cet appel et ce concept. La notion de pertinence correspond à notre heuristique décrite dans la section précédente. Le rapport peut ensuite être imprimé en partie ou en totalité. De même, chaque paragraphe (correspondant à une proposition particulière) peut également être sauvegardé en texte brut dans la base de connaissances.

\section{Cas d'utilisation}

Différents tests ont été menés pour évaluer le fonctionnement et la pertinence de notre plate-forme vis-à-vis de notre modèle théorique, ainsi que la qualité du support apporté aux décideurs d'une organisation. La brique de gestion de connaissances a été évaluée en milieu universitaire afin d'étudier sa réception auprès du public dans le cadre d'un travail collaboratif. Les résultats de cette étude sont présentés dans la section 5.1. Une seconde campagne d'expérimentations a été menée pour tester le comportement de la brique inférentielle et de la brique de visualisation. Cette seconde série d'évaluations a pris la forme d'entretiens auprès d'un expert et d'un chef de projets dont les résultats sont présentés dans la section 5.2.

\subsection{Utilisation de la brique de gestion de connaissances}

Le prototype que nous avons développé a été utilisé par des étudiants dans le cadre d'un cours enseigné à l'UTC. Durant quatre mois au semestre d'automne 2012, une quarantaine d'étudiants ont réalisé une veille technologique sur un sujet particulier. Il leur a été imposé dans un premier temps de définir le périmètre de leurs recherches au moyen d'une ontologie. Celle-ci permet de définir les notions clés identifiées pour effectuer leur veille. Dans un second temps il leur a été demandé d'utiliser la plateforme E-MEMORAe 2.0 pour collaborer, organiser et capitaliser au fil de l'eau le fruit de leurs recherches. À la fin du semestre un questionnaire leur a été donné, nous permettant d'obtenir des retours sur la manière dont ils avaient utilisé la plate-forme. Le questionnaire a la forme d'un formulaire en ligne. La participation à l'étude était libre et les réponses anonymes, aussi aucun entretien postérieur n'a été réalisé. Nous avons pu recueillir 21 témoignages. Les questions étaient de trois formes : échelle de Likert (échelle d'évaluation sur 5 valeurs, ici allant de 1 signifiant «pas du tout ou nul », à 5 signifiant «beaucoup ou très intéressant »), question à choix multiple où une seule réponse était autorisée et questions ouvertes.

Concernant les outils de la plate-forme E-MEMORAe 2.0, $48 \%$ des répondants ont utilisé le forum (réponses allant de 2 à 4), $52 \%$ le Wiki (réponses allant de 2 à 5) et $62 \%$ l'outil d'annotation réponse de 2 à 5). Ils sont par contre $71 \%$ à avoir utilisé beaucoup (5) un traitement de texte externe à la plate-forme et $62 \%$ à avoir utilisé un outil de cartographie mentale (Freemind, etc.) pour construire leur ontologie (réponses 
de 3 à 5). Un dernier champ libre leur permettait de nous faire connaître d'autres logiciels utilisés dans leur veille. La suite Google Docs en ressort très majoritaire.

Concernant les types de ressources partagées, les réponses ont été assez uniformes. Chacun des types proposés (articles académiques, articles encyclopédiques, articles d'opinions, support de présentations) a été capitalisé par au moins $40 \%$ des répondants avec une valeur de jugement de 4 ou 5 (nombreuses fois à beaucoup). Seuls les livres scientifiques (ressources peu pratiques à lire en quatre mois et à partager numériquement) et les comptes-rendus de réunion ont été très peu, voire pas du tout (score de 1 ou 2 ) capitalisés par plus de $40 \%$ des répondants. Ce dernier point peut s'expliquer par une absence fréquente de rédaction de compte-rendu.

Par ailleurs, les résultats montrent que les répondants ont très peu partagé de ressources entre différents groupes d'utilisateurs : $86 \%$ des répondants n'ont partagé leurs ressources que dans un seul groupe. L'indexation s'est révélée relativement pauvre : $67 \%$ des répondants déclarent n'avoir indexé des ressources que par un concept. Seuls $33 \%$ ont effectué une multi-indexation (au maximum par 3 concepts). La durée finalement assez courte du semestre explique peut-être qu'ils n'aient pas eu le temps ou l'opportunité de plus partager leurs ressources ou les indexer de manière plus large. Une étude supplémentaire sur une période plus longue permettrait de confirmer ou amender ce résultat.

Sur l'ergonomie générale de la plate-forme, son apparence actuelle a semblé trop éloignée de ce que les étudiants ont l'habitude d'utiliser. L'absence d'une vue globale sur un flux d'activité (comparable au «Wall» de Facebook ou Yammer, à la «Timeline» de Twitter) et l'obligation de passer exclusivement par la cartographie pour accéder aux ressources ont souvent déstabilisé les étudiants. Au moment de la mise à disposition de la plate-forme, cette dernière ne permettait pas par exemple d'avertir les utilisateurs si de nouvelles activités avaient lieu autour des thématiques qu'ils suivaient. Cette fonctionnalité de flux d'activité a depuis été ajoutée en permettant la mise en place d'alerte suite à l'indexation de ressources au sein de la plateforme, ainsi que la mise en place du service de minimessage tel que décrit dans la section 4. Ce dernier permet de retrouver un environnement de type « Wall » sur lequel les utilisateurs échangent des informations, sans pour autant négliger leur capitalisation.

Enfin la plupart des avis que nous avons pu recueillir étaient très favorables à l'utilisation d'un outil sémantique pour organiser un travail de réflexion et d'analyse tel que les étudiants avaient eu à réaliser. Les répondants sont ainsi $57 \%$ à trouver la navigation sur la cartographie de concept intéressante, $67 \%$ à apprécier le fait de pouvoir se constituer en groupe libre et $81 \%$ à apprécier pouvoir capitaliser différents types de ressources selon le même référentiel (scores de 4 ou 5).

\subsection{Utilisation de la brique inférentielle et de la brique de visualisation}

Nous avons testé ces deux briques auprès de deux collaborateurs de Thales R\&T. Nous avons présenté et testé la brique de visualisation, cette dernière utilisant directe- 
ment les heuristiques de la brique inférentielle et leurs résultats. Cette expérimentation a pris la forme d'un entretien oral avec chacune des deux personnes interrogées. Cet entretien a systématiquement eu lieu après une démonstration des différentes fonctionnalités de la plate-forme. Bien que la discussion au cours de l'expérimentation ait été très libre, toute l'expérience suivait un protocole établi à l'avance. Les personnes interrogées n'ont cependant jamais eu accès à ce protocole qui restait dans la main de l'interrogateur. Nous souhaitions, à l'aide de ces entretiens, valider la pertinence de l'outil et des heuristiques sous-jacentes dans :

- un travail de veille technologique et concurrentielle;

- un processus décisionnel lié à l'établissement d'une stratégie concurrentielle ;

- un processus décisionnel lié au montage de projet.

Nous avons alors identifié deux types de profils :

- le profil chef de projet, qui intervient lors de la phase de montage d'un projet cadre comme les projets européens, ANR, ou DGA et en particulier lors de l'établissement de la proposition de consortium;

- le profil expert technologique, qui intervient à différents niveaux dans la stratégie globale de l'établissement et dont les activités de veille technologique et concurrentielle sont primordiales pour l'organisation.

Le premier utilisateur interrogé est donc un chef de projet senior au sein du laboratoire de raisonnement et analyses dans les systèmes complexes et, à ce titre, monte et gère plusieurs projets en parallèle. Il est également souvent mobilisé lors de réunions de lancement d'appel à projets pour représenter les intérêts du laboratoire et identifier la pertinence de l'appel quant à ces mêmes intérêts. Le second entretien s'est déroulé, quant à lui, avec un expert technologique du laboratoire de raisonnement et analyses dans les systèmes complexes.

La suite de cette section est une synthèse des deux entretiens. L'expérimentation s'est déroulée en deux parties. Il s'agissait dans un premier temps de faire une démonstration de l'outil, avant que l'entretien en lui-même ne débute. L'ensemble de l'expérience devait se dérouler dans le bureau de l'utilisateur, sur son poste de travail, la brique de visualisation étant accessible à l'aide d'un simple navigateur web. Cependant, lors de l'entretien avec l'expert, une incompatibilité de version du navigateur internet est apparue et l'entretien s'est finalement effectué à l'aide de la machine utilisée pour le développement de la plate-forme.

La présentation de la brique de visualisation et de ses fonctionnalités a duré quarante minutes. Au cours de cette présentation, quelques bugs logiciels ont été remarqués ainsi que des problèmes ergonomiques. Entre autres, deux demandes particulières ont été formulées par le chef de projet pour améliorer la brique de visualisation et par extension les heuristiques mises en place. 
- Dans l'outil de génération de rapport de montage de consortium, présenter les organisations candidates sous la forme d'un graphe de collaboration et non sous la forme d'une liste.

- Modifier le comportement de la recherche et de la mise en valeur des concepts au sein du graphe de collaboration ou du cadran de suivi de tendance. L'heuristique actuelle cherche en effet à synthétiser au mieux la recherche formulée. Si l'utilisateur entre «fusion » par exemple, l'heuristique va agréger sous une seule thématique «fusion» toutes les informations indexées par «information fusion», «soft fusion» ou encore «radar fusion». Il est alors impossible de connaître en un coup d'œil les composants de cet agrégat, autrement qu'en procédant à un inventaire manuel en fonction des informations récupérées. L'idée est alors de présenter à l'utilisateur l'ensemble des concepts identifiés et les informations liées, sans les agréger. Une visualisation multifacettes (Cabanac et al., 2010) pourrait être intéressante dans ce cas.

La seconde partie de l'entretien a consisté en la prise en main de la brique de visualisation par le chef de projet interrogé. Cette prise en main était dirigée par l'intermédiaire de questions que l'interrogateur lui posait.

La première série de questions servait à mesurer la compréhension des différentes fonctionnalités de navigation dans le temps et dans l'espace au sein du cadran affichant le graphe des collaborations. Il s'agissait d'identifier des relations anciennes entre partenaires, des relations récentes mais fortes et enfin des relations régulières. La subtilité provenait du fait que ces trois types de relations pouvaient s'exprimer de prime abord sous la forme d'un lien plus fort entre deux partenaires et que seule l'utilisation du curseur de navigation dans le temps permettrait de différencier ces différents types de relations. Bien qu'ayant formellement identifié ces trois types de relation comme étant les liens les plus forts entre partenaires, le chef de projet n'a pas pu expliquer une méthode permettant d'identifier plus formellement chaque type de relation demandé. Il n'a pas utilisé le curseur de navigation temporel. À l'inverse, l'expert s'est souvenu de la tirette de navigation dans le temps et s'en est servi correctement pour identifier des relations anciennes ou régulières entre entités sur le graphe des collaborations.

Une seconde série de questions portait sur l'utilisation de la mise en évidence de thématiques et de partenaires dans le graphe. Cette série de questions n'a pas posé de problème particulier, le chef de projet ayant réussi du premier coup à mettre en valeur la thématique demandée et à nommer des partenaires ayant travaillé sur cette thématique, mais pas ensemble. La série de question concernant l'outil de génération de rapport de montage de consortium n'a pas posé de problème particulier.

La dernière série de questions devait permettre d'en connaître plus sur les habitudes de la personne interrogée. À la question de donner les sources habituelles permettant d'identifier de potentiels partenaires pour des projets technologiques ou commerciaux, le chef de projet nous a cité, par ordre d'importance :

1. les experts de son organisation;

2. les salons professionnels; 
3. les précédentes propositions de son organisation en réponse à des appels à projets;

4. les avis de différents partenaires connus;

5. les chambres de commerces et d'industries.

De son côté, l'expert nous a cité d'autres sources consultées régulièrement :

1. les fiches de renseignements mises à disposition par l'Union Européenne;

2. les publications scientifiques et les brevets déposés par les partenaires ou concurrents ;

3. les activités de réseautage de l'expert, entre autre grâce aux réunions de lancement des appels à projets.

L'entretien s'est terminé par une demande d'impression générale sur la plateforme, telle qu'elle avait été présentée, manipulée, ressentie. La principale impression du chef de projet interrogé est que cet outil répond bien à un souci qu'il rencontre régulièrement, à savoir pouvoir identifier un partenaire potentiel pour compléter un possible consortium dans le cadre d'une réponse à un appel à projet. En ce sens l'expérience a été très positive. Il regrette de ne plus pouvoir interagir avec le système pour entrer des paramètres supplémentaires afin d'affiner les entités présentées, que ce soit au sein du graphe des collaborations comme de l'outil de génération de rapport, par exemple pour n'observer que ses concurrents, ou qu'un type particulier de partenaire (PME, académique, etc.). Il a enfin pointé du doigt les faiblesses de l'outil et en particulier le manque d'informations concernant les calculs effectués ou les fonctionnalités disponibles qui ne favorisent pas la confiance dans les résultats affichés, du fait qu'il était difficile d'en comprendre la provenance. Une véritable analyse de l'utilisabilité de la plateforme sera nécessaire afin d'évaluer sa qualité (Nielsen, 1993 ; Shneiderman, 1997).

Le manque le plus criant relevé par l'expert, de son côté, sur les outils actuellement disponibles pouvant l'épauler dans sa veille sur les activités de ses domaines d'expertise est le manque d'automatisme dans la collecte des données. L'expert regrette que les outils ne soient pas capables de s'alimenter seuls, par exemple auprès des différents organismes ouvrant des appels à projets, afin de lui apporter les informations les plus fraîches. L'expert a en ce sens apprécié l'idée de la base de connaissances partagée par tous les membres de l'organisation permettant une meilleure couverture des actualités.

\section{Conclusion}

Nous avons vu dans cet article pourquoi il est intéressant de pouvoir capitaliser à la fois les ressources documentaires classiques et les ressources sociales, c'est-à-dire issues d'un processus social ou collaboratif, selon le même référentiel. Nous avons présenté un prototype de plate-forme de type écosystème numérique de connaissance permettant une telle capitalisation et la réutilisation des ressources produites lors de l'utilisation de la brique de gestion de connaissances comme support de décision au 
sein d'une organisation. L'ontologie que nous avons construite dans le cadre de ce projet permet en effet de représenter différents types de ressources et de les indexer par un même référentiel.

Le prototype a été utilisé comme support d'une veille technologique dans le cadre d'un cours à l'UTC. Cette expérimentation nous a permis d'identifier des voies d'améliorations possibles afin de faciliter les interactions des utilisateurs et leur collaboration. Ainsi, des fonctionnalités liées au suivi des activités des collaborateurs entre eux viennent d'être rendues disponibles. Néanmoins, les entretiens réalisés pour évaluer la brique de visualisation et à travers elle la brique inférentielle ont été très positifs. Les décideurs interrogés ont souligné l'intérêt de l'outil proposé pour permettre la mise en évidence de partenaires potentiels pour des projets collaboratifs. Cette mise en évidence est calculée à partir des données de tout ordre ajoutées à une base de connaissances.

Notre positionnement autour de la notion de document numérique et de ressource et la manière dont nous avons modélisé les différents types de ressources nous permettent d'utiliser toutes sortes de données. L'utilisation d'heuristiques conçues pour étudier des phénomènes sociaux sur différents types de données, dont celles issues de bases documentaires et bien sûr celles issues de médias sociaux, apporte des résultats intéressants quant à l'extraction d'une vue globale du réseau de collaboration d'une organisation et de son évolution dans le temps. Cette extraction, et sa mise en forme de manière à pouvoir être présentée à des décideurs, a fortement intéressé les décideurs interrogés qui y voient un moyen d'accéder facilement à de nouvelles connaissances qu'ils n'acquéraient auparavant qu'au prix d'un long travail manuel. Les retours obtenus lors de nos entretiens auprès des décideurs de Thales nous permettent de valider l'idée que les ressources issues des médias sociaux peuvent être sources de connaissances, en l'occurrence en apportant une vision claire sur un réseau d'organisations.

\section{Remerciements}

Ces travaux sont financés dans le cadre d'une thèse DGA-CIFRE.

\section{Bibliographie}

Barnes J. A. (1954). Class and committees in a norwegian island parish. Human Relations, vol. 7, p. 39-58.

Bojārs U., Breslin J. G. (2010). Semantically-interlinked online communities ontology. Consulté sur http://rdfs.org/sioc/spec/

Boughzala I., Dudezert A. (2012). Knowledge management 2.0: Organizational models and enterprise strategies. IGI Global.

Boyd D. M., Ellison N. B. (2008). Social network sites: Definition, history, and scholarship. Journal of Computer-Mediated Communication, vol. 13, n 1, p. 210-230.

Bray D. (2007). Knowledge ecosystems: A theoretical lens for organizations confronting hyperturbulent environments. In T. McMaster, D. Wastell, E. Ferneley, J. I. DeGross (Eds.), Or- 
ganizational dynamics of technology-based innovation: Diversifying the research agenda, p. $457-462$. Springer.

Breslin J. G., Bojārs U., Passant A., Fernández S., Decker S. (2009). Sioc: Content exchange and semantic interoperability between social networks. In W3c workshop on the future of social networking. Barcelona, España. Consulté sur http://www.w3.org/2008/09/msnws/ papers/sioc.html

Brickley D., Miller L. (2010). Foaf vocabulary specification. Consulté sur http://xmlns.com/ foaf/spec/

Cabanac G., Chevalier M., Chrisment C., Julien C. (2010, décembre). Organization of digital resources as an original facet for exploring the quiescent information capital of a community. International Journal on Digital Libraries, vol. 11, no 4, p. 239-261.

D’Arcus B., Giasson F. (2009). Bibo specifications. Consulté sur http://bibliontology.com/ specification

Deparis É. (2013). Création de nouvelles connaissances décisionnelles pour une organisation via ses ressources sociales et documentaires. Thèse de doctorat non publiée, Université de Technologie de Compiègne, Compiègne, France.

DiMicco J. M., Geyer W., Millen D. R., Dugan C., Brownholtz B. (2009). People sensemaking and relationship building on an enterprise social network site. In Proceedings of the 42nd hawaii international conference on system sciences, p. 1-10. Waikoloa, HI, USA, IEEE Computer Society.

Ehrlich K., Lin C.-Y., Griffiths-Fisher V. (2007). Searching for experts in the enterprise: Combining text and social network analysis. In Proceedings of the international acm conference on supporting group work, p. 117 -126. Sanibel Island, FL, USA, Association for Computing Machinery.

Ermine J.-C. (2000). La gestion des connaissances, un levier stratégique pour les entreprise. In P. Tchounikine (Ed.), Actes des 11es journées francophones d'ingénierie des connaissances. Toulouse, France. Consulté sur http://www.irit.fr/IC2000/ACTES/texte-ermine .$h t m l$

Ferreira A., Plessis T. du. (2009). Effect of online social networking on employee productivity. South African Journal of Information Management, vol. 11, n 1, p. 1-16.

Fisher D., Smith M., Welser H. T. (2006). You are who you talk to: Detecting roles in usenet newsgroups. In Proceedings of the 39th hawaii international conference on system sciences, vol. 3, p. 1 -10. Kauai, HI, USA, IEEE Computer Society.

Lainé-Cruzel S. (2004). Documents, ressources, données : les avatars de l'information numérique. Revue Information-Interaction-Intelligence, vol. 4, n $\mathrm{n}^{\circ}$, p. 105-119.

Leblanc A., Abel M.-H. (2008). A forum-based organizational memory as organizational learning support. International Journal of Digital Information Management, vol. 6, $\mathrm{n}^{\circ} 4$, p. 303-312.

Leblanc A., Abel M.-H. (2010). E-memorae2.0: A web platform dedicated to organizational learning needs. In Proceedings of the 3rd world summit on the knowledge society, wsks 2010, p. 306-315. Corfou, Grèce.

Lortal G., Laudy C., Deparis É., Mattioli J. (2013, novembre). Approaches for multi-granular fusion for social data analysis for a decision and intelligence application. In Proceedings of 
the 10th international conference on modeling decisions for artificial intelligence. Barcelona, España.

McPherson M., Smith-Lovin L., Cook J. M. (2001). Birds of a feather: Homophily in social networks. Annual Review of Sociology, vol. 27, p. 415-444.

Nielsen J. (1993). Usability engineering. San Francisco, CA, USA, Morgan Kaufmann Publishers Inc.

Nonaka I., Takeuchi H. (1995). The knowledge-creating company: how japanese companies create the dynamics of innovation. New York, NY, USA, Oxford University Press.

O'Reilly T. (2009, octobre). Web squared: Web 2.0 five years on. Rapport technique. San Francisco, Web 2.0 Summit. Consulté sur http://www.web2summit.com/web2009/public/ schedule/detail/10194

Prax J.-Y. (2012). Le manuel du knowledge management - mettre en réseau les hommes et les savoirs pour créer de la valeur ( $3^{\mathrm{e}}$ éd.). Dunod.

Pédauque R. T. (2003, juillet). Document : forme, signe et medium, les re-formulations du numérique. Consulté sur http://archivesic.ccsd.cnrs.fr/sic_00000511 (RTP-DOC (RTP 33 "Documents et contenu : création, indexation, navigation" - STIC - CNRS))

Seufert A., Krogh G. von, Back A. (1999). Towards knowledge networking. Journal of Knowledge Management, vol. 3, no 3, p. 180-190.

Shirky C. (2008). Here comes everybody - the power of organizing without organizations. Penguin Group.

Shneiderman B. (1997). Designing the user interface: Strategies for effective human-computer interaction (3rd éd.). Boston, MA, USA, Addison-Wesley Longman Publishing Co., Inc.

Tsai W., Ghoshal S. (1998, août). Social capital and value creation: The role of intrafirm networks. Academy of Management Journal, vol. 41, n 4, p. $464-476$.

Waltz E. (2003). Knowledge management in the intelligence enterprise. Artech House. 
